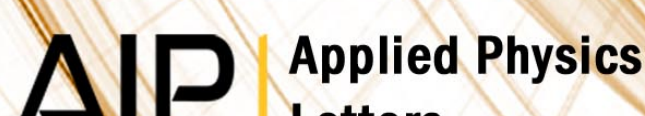 Letters
}

\section{Luminescence from two-dimensional electron gases in InAIN/GaN heterostructures with different In content}

M. F. Romero, M. Feneberg, P. Moser, C. Berger, J. Bläsing et al.

Citation: Appl. Phys. Lett. 100, 212101 (2012); doi: 10.1063/1.4720087

View online: http://dx.doi.org/10.1063/1.4720087

View Table of Contents: http://apl.aip.org/resource/1/APPLAB/v100/i21

Published by the American Institute of Physics.

\section{Related Articles}

Characteristics of indium incorporation in InGaN/GaN multiple quantum wells grown on a-plane and c-plane GaN Appl. Phys. Lett. 100, 212103 (2012)

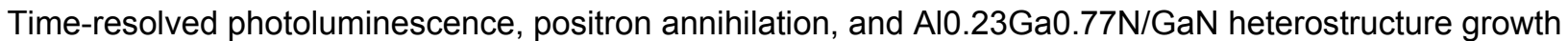
studies on low defect density polar and nonpolar freestanding GaN substrates grown by hydride vapor phase epitaxy

J. Appl. Phys. 111, 103518 (2012)

Modeling of carrier lifetimes in uniaxially strained GaAs

J. Appl. Phys. 111, 103704 (2012)

Observation of band alignment transition in InAs/GaAsSb quantum dots by photoluminescence J. Appl. Phys. 111, 104302 (2012)

The role of glass-viscosity on the growth of semiconductor quantum dots in glass matrices

J. Appl. Phys. 111, 094315 (2012)

\section{Additional information on Appl. Phys. Lett.}

Journal Homepage: http://apl.aip.org/

Journal Information: http://apl.aip.org/about/about_the_journal

Top downloads: http://apl.aip.org/features/most_downloaded

Information for Authors: http://apl.aip.org/authors

\section{ADVERTISEMENT}
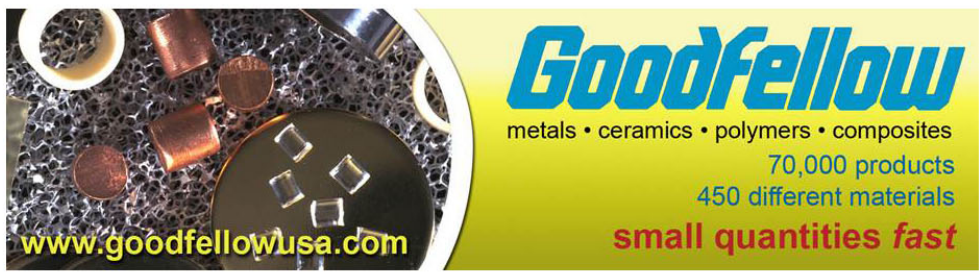


\title{
Luminescence from two-dimensional electron gases in InAIN/GaN heterostructures with different In content
}

\author{
M. F. Romero, ${ }^{1}$ M. Feneberg, ${ }^{1}$ P. Moser, ${ }^{1}$ C. Berger, ${ }^{1}$ J. Bläsing,${ }^{1}$ A. Dadgar,${ }^{1}$ A. Krost,${ }^{1}$ \\ E. Sakalauskas, ${ }^{2}$ and R. Goldhahn ${ }^{1,2}$ \\ ${ }^{1}$ Institut für Experimentelle Physik, Otto-von-Guericke-Universität Magdeburg, 39106 Magdeburg, Germany \\ ${ }^{2}$ Institut für Physik, Technische Universität Ilmenau, PF 100565, 98684 Ilmenau, Germany
}

(Received 3 April 2012; accepted 5 May 2012; published online 21 May 2012)

\begin{abstract}
The luminescence properties of $\operatorname{In}_{x} \mathrm{Al}_{1-x} \mathrm{~N} / \mathrm{GaN}$ heterostructures are investigated systematically as a function of the In content $(x=0.067-0.208)$. The recombination between electrons confined in the two-dimensional electron gas and free holes in the GaN template is identified and analyzed. We find a systematic shift of the recombination with increasing In content from about $80 \mathrm{meV}$ to only few meV below the GaN exciton emission. These results are compared with model calculations and can be attributed to the changing band profile and originating from the polarization gradient between InAlN and GaN. @ 2012 American Institute of Physics. [http://dx.doi.org/10.1063/1.4720087]
\end{abstract}

$\mathrm{In}_{x} \mathrm{Al}_{1-x} \mathrm{~N} / \mathrm{GaN}$ heterostructures (HSs), in particular for the lattice-matched composition $x \approx 0.18$, have emerged recently as promising candidates for next-generation electronic devices in millimeter- and submillimeter-wave applications $^{1,2}$ as well as for the improvement of optoelectronic devices. ${ }^{3,4}$ The use of InAlN layers offers several attractive advantages, such as a strong spontaneous polarization that induces an extremely high sheet carrier charge density in the two-dimensional-electron-gas (2DEG) (about $3.5 \times 10^{13} \mathrm{~cm}^{-2}$ for $14 \mathrm{~nm}$ thick $\mathrm{Al}_{0.93} \operatorname{In}_{0.07} \mathrm{~N}$ barriers), ${ }^{5,6}$ and a strain-free heterojunction that could reduce structural defects caused by the lattice mismatch. ${ }^{7,8}$ Moreover, due to their relatively high difference in refractive indices and the possibility of lattice matching, InAlN/GaN multilayer stacks with quarter-wave layers are a very promising approach for distributed Bragg mirrors ${ }^{9}$ and its application in nitride microcavity light-emitting diodes. ${ }^{10}$

Regardless of the final application, investigations on both the electrical and optical properties of the 2DEG are crucial for understanding the intrinsic properties of the InAlN/GaN heterostructures, which is the bottleneck to advance the nitride electronics technology. Up to now, InAlN/GaN HS have been studied widely by electrical measurements, ${ }^{5,11}$ however, there is still a lack of systematic studies concerning optical characterization.

In this work, we present photoluminescence (PL) as a method to investigate the influence of the In content in InAlN/GaN HS on the band shape and the energy levels in the 2DEG. A 2DEG-related luminescence with a strong dependence on the In content was identified. Furthermore, model calculations were performed correlating the experimental results to the recombination of electrons in the 2DEG located in an excited state and free holes in the GaN buffer layer.

A set of nine $\operatorname{In}_{x} \mathrm{Al}_{1-x} \mathrm{~N} / \mathrm{GaN}$ HS samples with similar layer thickness but different In content, varying from $x=0.067$ to 0.208 as described in Table $\mathrm{I}$, were analyzed systematically. The HS with metal face (0001) orientation were grown by metal organic vapour phase epitaxy (MOVPE) in an AIXTRON 200/4 RF-S reactor on 2" c-plane sapphire substrates with a nominal miscut of $0.25^{\circ}$ towards the (1100)- plane. Standard precursors (trimethylaluminum $\left[\left(\mathrm{CH}_{3}\right)_{3} \mathrm{Al}\right.$ : TMAl], trimethylindium $\left[\left(\mathrm{CH}_{3}\right)_{3} \mathrm{In}\right.$ : TMIn], trimethyl-gallium $\left[\left(\mathrm{CH}_{3}\right)_{3} \mathrm{Ga}\right.$ : TMGa] and ammonia $\left.\left(\mathrm{NH}_{3}\right)\right)$ were used. The growth of the $\mathrm{In}_{x} \mathrm{Al}_{1-x} \mathrm{~N}$ layer took place at temperatures in the range from $\mathrm{T}=900{ }^{\circ} \mathrm{C}$ (for $x=0.067$ ) to $\mathrm{T}=740^{\circ} \mathrm{C}$ (for $x=0.208$ ), and the growth rate $\sim 0.1 \mu \mathrm{m} / \mathrm{h}$. The GaN layer was grown at $1145^{\circ} \mathrm{C}$ for each $\mathrm{HS}$, and the growth temperature of the AlN seed layer on top of the substrate was $685^{\circ} \mathrm{C}$, except for two samples (with an In content of $18.5 \%$ and $19.0 \%$, respectively) that was $950^{\circ} \mathrm{C}$. All layers were grown without additional doping. Hall studies yielded a residual electron concentration for the GaN buffer layer of $2 \times 10^{15} \mathrm{~cm}^{-3}$. The formation of the 2DEG did not allow to investigate the electrical properties of the thin InAIN films.

Both the thicknesses of the InAlN layer and the lattice parameters $c(\mathrm{GaN})$ corresponding to the out-of-plane lattice parameter of the GaN buffer layer were measured by high resolution x-ray diffraction (HR-XRD). The in-plane lattice parameters of the surface $a$ (InAlN surface) were measured by grazing incidence in-plane diffraction (GIID). On the other hand, HR-XRD was also used to determine the In content with an accuracy of $\pm 0.5 \%$. In Table I, the values of $\varepsilon_{z z}$ in $\mathrm{GaN}$ were calculated from the $c$-lattice parameters assuming the relaxed value of $c_{0}(\mathrm{GaN})=5.18523 \AA$ given by Ref. 12. It shows compressive biaxial strain (i.e., $\varepsilon_{z z}(\mathrm{GaN})>0$ )

TABLE I. Overview of the samples sorted by the In content. The thickness of the top layer, the strain of GaN buffer layer $(1.5 \mu \mathrm{m})$ in the growth direction [0001] and the $a$-lattice parameter of the InAlN surface are shown.

\begin{tabular}{lcccc}
\hline \hline$[\mathbf{I n}](\boldsymbol{\%})$ & InAlN thickness $(\mathrm{nm})$ & $\varepsilon_{z z}(\mathrm{GaN}) \times 10^{-4}$ & \multicolumn{2}{c}{$a(\operatorname{InAlN}$ surface $)(\AA)$} \\
\hline $\mathbf{6 . 7}$ & 33.2 & 5.34 & 3.1775 & Partially relaxed \\
$\mathbf{8 . 5}$ & 33.4 & 4.18 & 3.1740 & Partially relaxed \\
$\mathbf{1 4 . 5}$ & 35.0 & 4.57 & 3.1852 & Strained \\
$\mathbf{1 7 . 6}$ & 33.4 & 5.34 & 3.1855 & Strained \\
$\mathbf{1 8 . 5}$ & 44.0 & 11.51 & 3.1805 & Strained \\
$\mathbf{1 9 . 0}$ & 45.7 & 8.42 & 3.1829 & Strained \\
$\mathbf{1 9 . 5}$ & 33.8 & 4.18 & 3.1856 & Strained \\
$\mathbf{2 0 . 5}$ & 30.6 & 4.18 & 3.1855 & Strained \\
$\mathbf{2 0 . 8}$ & 29.6 & 5.34 & 3.1849 & Strained \\
\hline \hline
\end{tabular}


of the templates, as expected from the mismatch of the thermal expansion coefficients with the sapphire substrates. ${ }^{13,14}$ The higher values of $\varepsilon_{z z}(\mathrm{GaN})$ for samples with $[\mathrm{In}]=18.5$ and $19.0 \%$ are explained by the different growth temperature of the AlN seed layer used for these HS in respect to the rest of the samples.

In addition, the $a$ (InAlN surface) parameters were compared with the $a$-lattice parameter in $\mathrm{GaN}$ that were calculated from the $c(\mathrm{GaN})$ values. The results are summarized in Table I and show that the InAlN layers with the lowest In content are partially relaxed, in contrast to the samples with $[\mathrm{In}] \geq 14.5 \%$ that are under strain. These results are in good agreement with previous studies where InAlN/GaN HS with In content between $13 \%$ and $32 \%$ were fully strained but samples with lower In content were partially relaxed. ${ }^{15}$

As reference sample, a GaN template without InAlN cap was also analyzed. For optical characterization by PL, a HeCd laser $(\lambda=325 \mathrm{~nm})$ was used as excitation source. The sample was placed in a liquid helium cryostat allowing variable temperatures between $5 \mathrm{~K}$ and room temperature (RT). Emitted light was dispersed by a grating monochromator with $1.25 \mathrm{~m}$ focal length equipped with a $1200 \mathrm{rules} / \mathrm{mm}$ grating, and PL spectra were recorded by a liquid nitrogen cooled CCD camera. The chosen spectral resolution was always better than $0.7 \mathrm{meV}$ at $3.5 \mathrm{eV}$.

Temperature dependent PL spectra of the $\operatorname{In}_{x} \mathrm{Al}_{1-x} \mathrm{~N} /$ GaN HS in the range from $5 \mathrm{~K}$ to RT were measured for all nine samples. Exemplarily, the evolution of the PL spectra for the sample with $x=0.176$ being nearly lattice matched to $\mathrm{GaN}$ is presented in Fig. 1. The expected recombination features of the GaN buffer are visible: the donor bound exciton $\left(\mathrm{D}^{0} \mathrm{X}\right.$ ) is located at $\sim 3.481 \mathrm{eV}$ (at $5 \mathrm{~K}$ ) dominating the luminescence at low temperatures. The energy position of the $\left(\mathrm{D}^{0} \mathrm{X}\right)$ is similar for all the analysed HS samples. Its position is blue-shifted by approximately $10 \mathrm{meV}$ in respect to that of

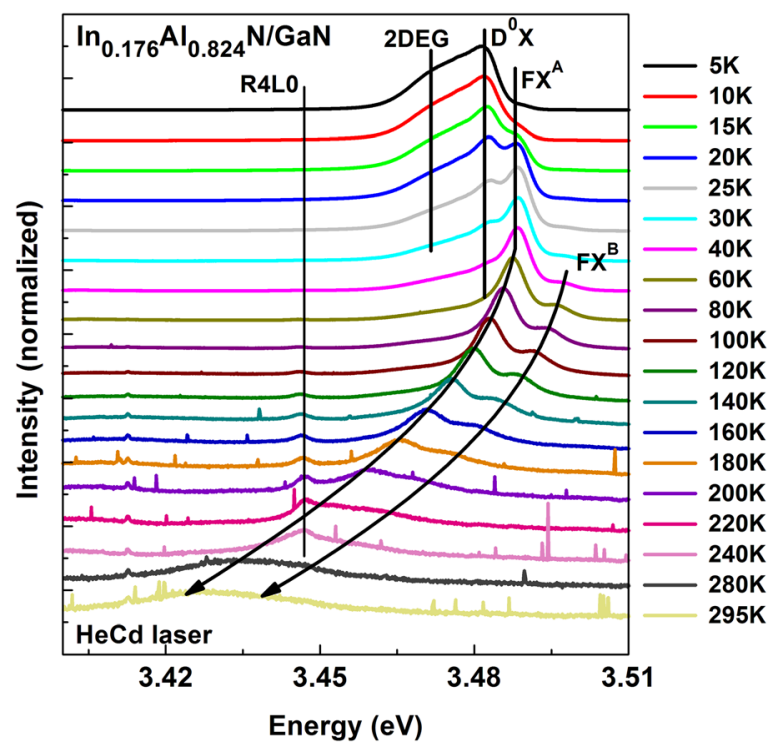

FIG. 1. Temperature dependent PL spectra of $\operatorname{In}_{0.176} \mathrm{Al}_{0.824} \mathrm{~N} / \mathrm{GaN}$ HS in the range of the GaN band gap between 5 and 295 K.The main features are identified as donor bound exciton $\left(\mathrm{D}^{0} \mathrm{X}\right)$, free excitons of $\mathrm{A}$ and $\mathrm{B}$ valence band $\left(\mathrm{FX}^{\mathrm{A}}\right.$ and $\mathrm{FX}^{\mathrm{B}}$ ), and related to the 2DEG. The peak at $\sim 3.445 \mathrm{eV}$ is identified as the fourth longitudinal optical phonon Raman line (R4LO) of the $325 \mathrm{~nm} \mathrm{He}-\mathrm{Cd}$ excitation laser source. unstrained GaN. This is in line with results from HR-XRD in Table I discussed previously. The free A exciton $\left(\mathrm{FX}^{\mathrm{A}}\right)$, located $7 \mathrm{meV}$ above the $\left(\mathrm{D}^{0} \mathrm{X}\right)$ becomes dominant and redshifts as the temperature increases, ${ }^{16}$ also the free $\mathrm{B}$ exciton $\left(\mathrm{FX}^{\mathrm{B}}\right)$, located $\sim 8 \mathrm{meV}$ above $\mathrm{FX}^{\mathrm{A}}$, is observable at elevated temperatures. The evolution of these two peaks as a function of temperature is marked by arrows in Fig. 1.

The PL spectrum at $\mathrm{T}=5 \mathrm{~K}$ shows an additional peak (labelled 2DEG in Fig. 1), slightly lower in energy than the excitonic features of GaN. Temperature dependent PL measurements reveal that the intensity of this additional 2DEGrelated peak quenches at temperatures at about $60 \mathrm{~K}$. In fact, similar contributions were observed previously for $\mathrm{AlGaN} /$ GaN HS (Refs. 17 and 18) and in $\mathrm{ZnMgO} / \mathrm{ZnO}$ HS (Ref. 19) that were assigned to the recombination of electrons in the 2DEG and free holes in the buffer layer.

The assignment of the additional peak as 2DEG related luminescence is corroborated by comparing the PL spectra of samples with different In content as shown in Fig. 2 for $\mathrm{T}=5 \mathrm{~K}$. The estimated peak energies of the $2 \mathrm{DEG}$-related recombination bands are marked by solid triangles. We observe a systematic shift of this band to higher energies when the In content of the top layer is increased, while the positions of the $\mathrm{FX}^{\mathrm{A}}$ and $\mathrm{D}^{0} \mathrm{X}$ peaks remain nearly constant and are only dependent on the strain in the buffer layer. Moreover, the 2DEG-related transition is not observed in the reference sample consisting of a $\mathrm{GaN}$ template only (see bottom trace in Fig. 2). Lower indium concentration in the cap layer leads to higher band offsets, a larger polarization gradient, and thus a stronger confinement of electrons. Therefore, the assignment of this spectral contribution to the presence of the 2DEG is straight forward, however, the nature of the luminescence mechanism remains to be understood.

Model calculations of the band structure and the quantized energy states in our samples were performed by solving the Schrödinger and Poisson equations self-consistently by using nextnano software. ${ }^{20}$ In the calculation, parameters for $\mathrm{GaN}$ and linear interpolations between the InN and AlN parameters for InAlN were used after Ref. 21, except the

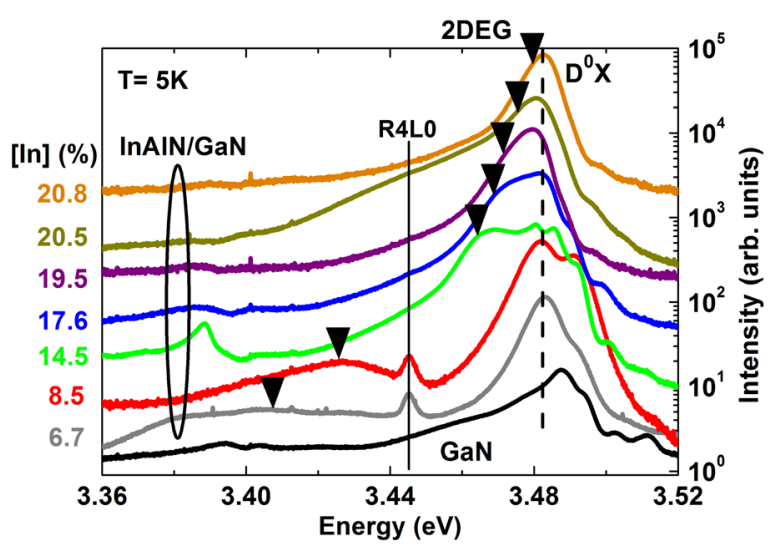

FIG. 2. Evolution of the PL emission of the InAlN/GaN HS in the range of the GaN bandgap at $5 \mathrm{~K}$, as a function of In content, in comparison with GaN template used as a reference. The peak at $\sim 3.445 \mathrm{eV}$ is identified as the fourth Raman line (R4LO) due to the $325 \mathrm{~nm}$ He-Cd excitation laser source. Peak energies of the 2DEG-related recombination bands are marked by solid triangles. 
bandgap energy of InAlN which was estimated by applying the empirical expression (13) from Ref. 22

$$
E_{A}^{A I I n N}=E_{A}^{I n N} \cdot x+E_{A}^{A I N} \cdot(1-x)-b_{2}(x) \cdot(1-x) \cdot x,
$$

where $b_{2}(x)=\mathrm{A} /\left(1+\mathrm{C} \cdot x^{2}\right)$ is the bowing parameter with $\mathrm{A}=6.43 \pm 0.12 \mathrm{eV}$ and $\mathrm{C}=1.21 \pm 0.14 \mathrm{eV}$ and $E_{A}^{I n N}$ $=0.675 \mathrm{eV}$ (Ref. 23) and $E_{A}^{A l N}=6.015 \mathrm{eV}$ (Refs. 24 and 25) at RT. Note that the input data are given at RT and the corrections due to the temperature change are taking into account in the calculations. Moreover, the use of the bandgap values is supported by complementary experimental results in some of the samples by using ellipsometry (not shown). The valence band order of the InAlN in our range of In composition is known to be $\Gamma_{7}-\Gamma_{9}-\Gamma_{7}{ }^{26}$

The surface potential for each HS was assumed to be given by

$$
e \phi_{b}=[0.5 \cdot x+2.1 \cdot(1-x)] \mathrm{e} V,
$$

where $x$ is the In content. The dependence was originally suggested for Ni Schottky contacts. ${ }^{21}$ It can be also applied here because the values for $\varphi_{b}$ are almost consistent to results of angle resolved x-ray photoelectron spectroscopy (XPS) at the free surfaces. ${ }^{27}$ In addition, XPS measurements $^{28}$ for thin films with $\mathrm{x}=0.17$ and 0.25 yielded 1.9 and $1.5 \mathrm{eV}$ while Eq. (2) corresponds to 1.83 and $1.7 \mathrm{eV}$, respectively. It is worth mentioning that these small differences in the barrier height lead due to the large barrier thickness (low barrier field strength) only to minor changes in the band profile.

As a first approximation, pseudomorphic HS are assumed and the values of the strain of the GaN buffer, calculated from the experimental values of the lattice constants, were used in the simulation. Under these assumptions, three energy levels are occupied in the 2DEG, i.e., lower in energy than the Fermi level $E_{F}$. The evolution of the calculated energy levels as a function of the In content for our samples is shown in Fig. 3. A schematic drawing of the band diagram of the heterostructure and the probability density function $|\psi|^{2}$ for the corresponding energy levels $\left(E_{n=1}, E_{n=2}\right)$

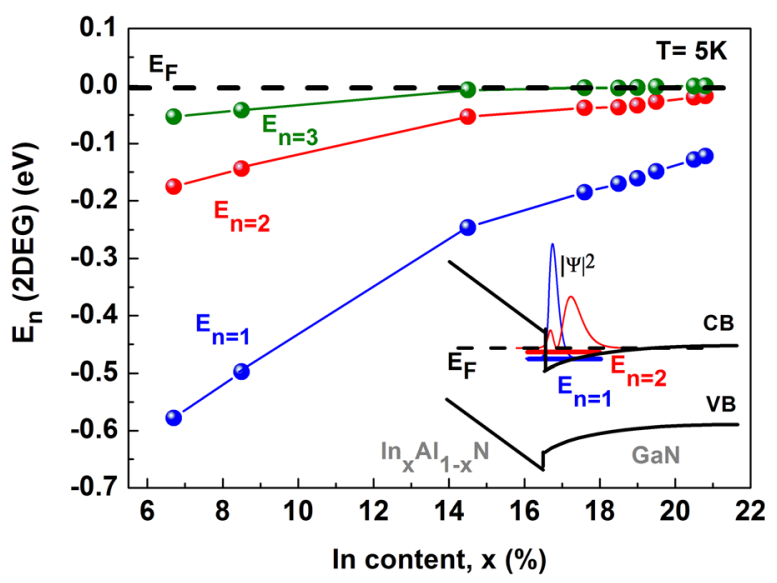

FIG. 3. Evolution of the occupied energy states in the 2DEG respect to the Fermi level $\left(\mathrm{E}_{\mathrm{F}}\right)$, as a function of the In content. Inset: schematic band diagram of the InAlN/GaN showing the probability function $|\psi|^{2}$ for $\mathrm{E}_{\mathrm{n}=1}$ and $\mathrm{E}_{\mathrm{n}}=2$. respect to $\mathrm{E}_{\mathrm{F}}$ (located close to the band edge) is presented in the inset of Fig. 3. The third occupied level $\left(E_{n=3}\right)$ is nearly at $\mathrm{E}_{\mathrm{F}}$ and it is not taken into account further in the current analysis.

As shown in Fig. 3 (inset), the electrons in the ground state are strongly localized in the quasi triangular quantum well, hence, far from the free holes in the GaN buffer, that are relaxing towards the highest energy position of the valence band (flatband region) due to band bending. In contrast, the location of electrons from the first excited state penetrates further into the GaN template leading to a higher recombination probability with the excited holes. In fact, a good agreement between the experimental 2DEG-related transition energy $\left(\mathrm{E}_{2 \mathrm{DEG}}\right)$ and the calculated first excited quantized state $\left(E_{n=2}\right)$ respect to the flatband energy position of the valence band in $\mathrm{GaN}\left(\mathrm{E}_{\mathrm{VB}, \max }\right)$ is observed, especially for the HS with $0.145 \leq x \leq 0.208$ (Fig. 4). It is worth mentioning that the HS with In content between $14.5 \%$ and $20.8 \%$ are fully strained as discussed previously and our simulation agrees with the experimental data within a deviation lower than $0.4 \%$. On the other hand, the $\operatorname{In}_{x} \mathrm{Al}_{1-x} \mathrm{~N}$ layers with $x=0.067$ and $x=0.085$ are partially relaxed, ${ }^{15}$ hence supporting the idea that the experimental luminescence energies are located between the calculated energy positions for strained and fully relaxed HS, as shown in Fig.4.

Therefore, these results highlight that the recombination of electrons from the 2DEG at the first excited level with photoexcited holes in the GaN is the most likely mechanism behind the 2DEG related PL emission, which is in good qualitative agreement with the results from J. P. Bergman et al. in $\mathrm{AlGaN} / \mathrm{GaN}$ HS. ${ }^{18}$ For higher indium concentration in the cap layer, the probability density of electrons reaches further into the $\mathrm{GaN}$ buffer which is mirrored by a more intense recombination signal in our spectra.

In conclusion, InAlN/GaN HS with different In contents have been systematically analysed by PL measurements for temperatures between $5 \mathrm{~K}$ and RT. We found a luminescence peak associated to the recombination between electrons in the 2DEG at the second level $\left(E_{n=2}\right)$ and photoexcited holes in the GaN buffer. Our findings are supported by the good correlation between the experimental transition energies and

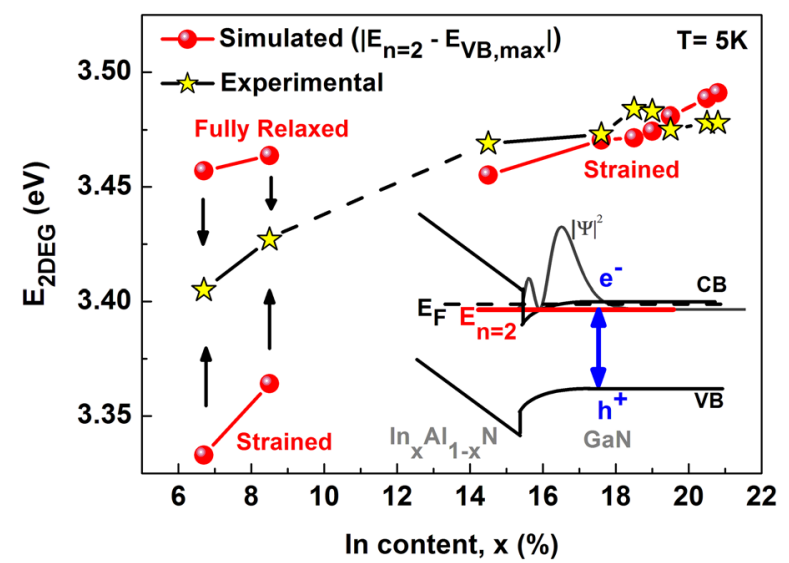

FIG. 4. Evolution of 2DEG-related transition energy, experimental (stars) and simulated (solid dots) for fully relaxed and strained InAIN layer, as a function of the In content. Inset: schematic band diagram of the InAlN/GaN showing the probability function $|\psi|^{2}$ for $\mathrm{E}_{\mathrm{n}=2}$ and the likely electron ( $\mathrm{e}^{-}$)hole $\left(\mathrm{h}^{+}\right)$interaction. 
model calculations. The dependence of the 2DEG-related emission on the In content can be understood with the changing band profile attributed to the different polarization gradient between InAIN and GaN.

This work was supported by the EU within the 7th RTD Framework (project RAINBOW: Contract No. PITN-GA2008-213238). Part of the work was supported by the German Science Foundation (DFG) in the framework of SFB 787. We also thank B. Garke and R. Metzner for providing the results of photo-electron spectroscopy.

${ }^{1}$ D. S. Lee, J. W. Chung, H. Wang, X. Gao, S. Guo, P. Fay, and T. Palacios, IEEE Electron Device Lett. 32, 755 (2011).

${ }^{2}$ M. Mikulics, R. Stoklas, A. Dadgar, D. Gregušová, J. Novák, D. Grützmacher, A. Krost, and P. Kordoš, Appl. Phys. Lett. 97, 173505 (2010).

${ }^{3}$ Z. T. Chen, S. X. Tan, Y. Sakai, and T. Egawa, Appl. Phys. Lett. 94, 213504, (2009).

${ }^{4}$ R. Butté, J.-F. Carlin, E. Feltin, M. Gonschorek, S. Nicolay, G. Christmann, D. Simeonov, A. Castiglia, J. Dorsaz, H. J. Buehlmann, S. Christopoulos, G. Baldassarri Höger von Högersthal, A. J. D Grundy, M. Mosca, C. Pinquier, M. A. Py, F. Demangeot, J. Frandon, P. G. Lagoudakis, J. J. Baumberg, and N. Grandjean, J. Phys. D: Appl. Phys. 40, 6328 (2007).

${ }^{5}$ M. Gonschorek, J.-F. Carlin, E. Feltin, M. A. Py, N. Grandjean, V. Darakchieva, B. Monemar, M. Lorenz, and G. Ramm, J. Appl. Phys. 103, 093714 (2008).

${ }^{6}$ D. Cavalcoli, S. Pandey, B. Fraboni, and A. Cavallini. Appl. Phys. Lett. 98, 142111 (2011).

${ }^{7}$ J.-F. Carlin and M. Ilegems, Appl. Phys. Lett. 83, 668 (2003).

${ }^{8}$ Ž. Gačević, S. Fernández-Garrido, J. M. Rebled, S. Estradé, F. Peiró, and E. Calleja, Appl. Phys. Lett. 99, 031103 (2011).

${ }^{9}$ A. Krost, C. Berger, J. Bläsing, A. Franke, T. Hempel, A. Dadgar, and J. Christen, Appl. Phys. Lett. 97, 181105 (2010).

${ }^{10}$ J. Dorsaz, J.-F. Carlin, S. Gradecak, and M. Ilegems, J. Appl. Phys. 97, 084505 (2005).

${ }^{11}$ A. Dadgar, F. Schulze, J. Bläsing, A. Diez, A. Krost, M. Neuburger, E. Kohn, I. Daumiller, and M. Kunze, Appl. Phys.Lett. 85, 5400 (2004).
${ }^{12}$ V. Darakchieva, B. Monemar, and A. Usui, Appl. Phys. Lett. 91, 031911 (2007).

${ }^{13}$ H. Amano, K. Hiramatsu, and I. Akasaki, Jpn. J. Appl. Phys. 27, L1384 (1988).

${ }^{14}$ C. Kisielowski, J. Krüger, S. Ruvimov, T. Suski, J. W. Ager III, E. Jones, Z. Liliental-Weber, M. Rubin, E. R. Weber, M. D. Bremser, and R. F. Davis, Phys. Rev. B 54, 17745-17753 (1996).

${ }^{15}$ C. Hums, J. Bläsing, A. Dadgar, A. Diez, T. Hempel, J. Christen, A. Krost, K. Lorenz, and E. Alves, Appl. Phys. Lett. 90, 022105 (2007).

${ }^{16}$ B. Monemar, I. A. Buyanova, J. P. Bergman, H. Amano, and I. Akasaki, Mater. Sci. Eng. B 43, 172 (1997).

${ }^{17}$ G. Martínez-Criado, C. Miskys, U. Karrer, O. Ambacher, and M. Stutzmann, Jpn. J. Appl. Phys. 43, 3360 (2004).

${ }^{18}$ J. P. Bergman, T. Lundström, B. Monemar, H. Amano, and I. Akasaki, Appl. Phys. Lett. 69, 3456 (1996).

${ }^{19}$ H. Chen, S. Gu, J. Liu, J. Ye, K. Tang, S. Zhu, and Y. Zheng, Appl. Phys. Lett. 99, 211906 (2011).

${ }^{20}$ nextnano, Suedmaehrenstr. 21, 85586 Poing, Germany. The nextnano simulation software can be obtained from: http://www.wsi.tum.de/nextnano3 and http://www.nextnano.de.

${ }^{21}$ O. Ambacher, J. Majewski, C. Miskys, A. Link, M. Hermann, M. Eickhoff, M. Stutzmann, F. Bernardini, V. Fiorentini, V. Tilak, B. Schaff, and L. F. Eastman, J. Phys.: Condens. Matter 14, 3399 (2002).

${ }^{22}$ E. Sakalauskas, H. Behmenburg, C. Hums, P. Schley, G. Rossbach, C. Giesen, M. Heuken, H. Kalisch, R. H. Jansen, J. Bläsing, A. Dadgar, A. Krost, and R. Goldhahn, J. Phys. D: Appl. Phys. 43, 365102 (2010).

${ }^{23}$ P. Schley, R. Goldhahn, G. Gobsch, M. Feneberg, K. Thonke, X. Wang, and A. Yoshikawa, Phys. Status Solidi B 246, 1177 (2009).

${ }^{24}$ M. Feneberg, R. A. R. Leute, B. Neuschl, K. Thonke, and M. Bickermann, Phys. Rev. B 82, 075208 (2010).

${ }^{25}$ G. Rossbach, M. Feneberg, M. Röppischer, C. Werner, N. Esser, C. Cobet, T. Meisch, K. Thonke, A. Dadgar, J. Bläsing, A. Krost, and R. Goldhahn, Phys. Rev. B 83, 195202 (2011).

${ }^{26}$ M. R. Laskar, T. Ganguli, A. A. Rahman, A. Arora, N. Hatui, M. R. Gokhale, S. Ghosh, and A. Bhattacharya, Appl. Phys. Lett. 98, 181108 (2011).

${ }^{27}$ R. Metzner and B. Garke "Surface band-bending of InAlN/GaN heterostructures determined by x-ray photoelectron spectroscopy" (unpublished).

${ }^{28}$ M. Akazawa, B. Gao, T. Hashizume, M. Hiroki, S. Yamahata, and N. Shigekawa, J. Appl. Phys. 109, 013703 (2011). 